\title{
Age and Life Course Location as Interpretive Resources for Decisions Regarding Disclosure of HIV to Parents and Children: Findings from the HIV and Later Life Study
}

Dana Rosenfeld, Damien Ridge, Jose Catalan, and Valerie Delpech, on behalf of the HIV and Later Life (HALL) team ${ }^{\mathrm{i}}$

ACCEPTED FOR PUBLICATION IN THE JOURNAL OF AGING STUDIES JUNE 8, 2016

ACKNOWLEDGEMENTS: The HIV and Later Life team thank the project's funders (the Lifelong Health and Wellbeing Cross-Council Programme), the older people living with HIV who participated in the study, and the clinics and HIV organizations that supported the study.

\section{Research highlights:}

Research oversimplifies the role of age in HIV disclosure

We explore how older people decide to disclose their HIV to children and parents

Participants treat age as a proxy for HIV knowledge and response to disclosure

This 'lifecoursing' reflects a more complex engagement with age than that captured by previous research into HIV, aging, and disclosure 


\begin{abstract}
Studies of disclosure among older people living with HIV (PLWH) are uninformed by critical socialgerontological approaches that can help us to appreciate how older PLWH see and treat age as relevant to disclosure of their HIV status. These approaches include an ethnomethodologicallyinformed social constructionism that explores how 'the' life course (a cultural framework depicting individuals' movement through predictable developmental stages from birth to death) is used as an interpretive resource for determining self and others' characteristics, capacities, and social circumstances: a process Rosenfeld and Gallagher (2002) termed 'lifecoursing'. Applying this approach to our analysis of 74 life-history interviews and three focus groups with older (aged 50+) people living with HIV in the United Kingdom, we uncover the central role that lifecoursing plays in participants' decision-making surrounding disclosure of their HIV to their children and/or older parents. Analysis of participants' accounts uncovered four criteria for disclosure: the relevance of their HIV to the other, the other's knowledge about HIV, the likelihood of the disclosure causing the other emotional distress, and the other's ability to keep the disclosed confidential. To determine if these criteria were met in relation to specific children and/or elders, participants engaged in lifecoursing, evaluating the other's knowledge of HIV, and capacity to appropriately manage the disclosure, by reference to their age. The use of assumptions about age and life-course location in decision-making regarding disclosure of HIV reflects a more nuanced engagement with age in the disclosure decision-making process than has been captured by previous research into HIV disclosure, including on the part of people aging with HIV.
\end{abstract}

Keywords: HIV, disclosure, stigma, life course 


\section{INTRODUCTION}

At the outset of the HIV/AIDS epidemic in the early 1980s, disclosure of HIV status in the West was an almost exclusive challenge to men who have sex with men (MSM), who were the most affected by the virus. But the diversification of the HIV/AIDS epidemic along lines of sex, sexual orientation, ethnicity, and nationality has introduced the necessary 'information management' of stigmatized identities (Goffman, 1963) to heterosexual men and women across the globe. The HIV population has further diversified along lines of age, as the emergence of successful antiretroviral medications in the mid-1990s changed HIV from a life-threatening to a manageable medical condition, and enables people living with HIV (PLWH) to survive into later life (van Sighem, Gras, Reiss, Brinkman \& de Wolf, 2010; Justice 2010; Nakagawa, May, \& Phillips 2013). This aging of the HIV population, bolstered by rising rates of HIV acquisition at older ages (Cresswell \& Fisher 2013; Greysen et al. 2013; Smith et al. 2011), introduces yet another layer of complexity to stigma management, as increasing numbers of older PLWH now manage the virus's medical, social, and psychological dimensions from the vantage point and social circumstances of older age (as, for example, parents and grandparents and the children of elderly parents). As with younger PLWH (Thoth, Tucker, Leahy \& Stewart 2014; Fernet et al. 2011; Fair 2012), living with HIV had at its centre dilemmas and strategies related to disclosure of HIV status - a process complicated by fear of rejection (Gilbert \& Walker 2010) and/or causing distress to the disclosed-to person (Rouleau, Côté \& Cara 2012; Derlaga et al. 2002; Petrak et al. 2011), and discrimination (Liamputtong 2013; Petrak et al. 2001) and even violence (Brown, Serovich, \& Kimberly 2016; Kennedy et al. 2015).

Yet research into disclosure practices amongst older PLWH remains relatively thin, and tends to replicate the tendency of most other studies of disclosure of HIV and of aging with HIV to, in Sankar et al’s (2011: 1188) words, conceptualise ‘age as a demographic variable only’ (by, for example, asking such questions as 'does the age of the PLWH and/or the age of the potential recipient of disclosure affect disclosure decisions, practices, and outcomes?'). Thus, while studies of how young or middle-aged parents living with HIV disclose their status to their young children (see e.g. Liamputtong \& Haritavorn 2014; McDonald 2013; Delaney, Serovich \& Lim 2008) have uncovered the centrality of their children's age to the decision-making process, the various uses of age and life course location as interpretive frames for decision-making regarding disclosure remain either wholly unconsidered or, at best, under-theorized. This gap in knowledge is exacerbated by the lack of research into disclosure by older PLWH of their HIV status to elderly parents (but see Shehan et al. 2005).

We argue that this represents a missed opportunity to make use of social-gerontological constructionist approaches that view age and aging in more dynamic ways and that can help us to identify the subjective relevance of age and life course location to disclosure of HIV status in later life. Our analysis of focus groups and life-history interviews that we conducted with older PLWH uncovered significant concerns over HIV stigma and disclosure to sexual and romantic partners, friends, and family (see Rosenfeld et al. 2015). Although participants' sense that age affected how stigma and disclosure affected their own lives as older PLWH was evident in accounts of disclosure to this range of others, it was in accounts of disclosure to parents and children that participants' use of the life course as an interpretive frame for disclosure decisions was most noticeable. As we will show, participants' decisions to disclose to children and/or older parents involve careful consideration of their children's and parents' historical exposure to information about HIV, on the one hand, and of their age and life course location as proxies for both their ability to understand and 
cope with the disclosed information and their likelihood of learning of their parent's HIV status from others, on the other. These interlinked assumptions about how others' circumstances, capacities, needs and exposure to new information are distributed across the life course when engaging in decision-making regarding disclosure demonstrate a more complex role of age in disclosure in later life than has been captured by previous research.

\section{THEORETICAL APPROACH: THE LIFE COURSE VERSUS 'LIFECOURSING’}

Dominant thinking about the life course found in wider cultural constructions and in such ahistorically-minded disciplines as developmental psychology depicts aging as a movement through discrete sequential stages of development. This depiction constructs these stages as reflecting innate age-based capacities and characteristics, with middle adulthood positioned, along an 'up' and 'down' hill representing the life course (Hockey \& James 1993), at the apex of human development, capacity/productivity, and integrity/power (Holstein \& Gubrium 2000, 2007; Rosenfeld and Gallagher 2002).

In contrast to this dominant view of life course stages, transitions, etc. as objective realities impacting individuals as they move across time, critical gerontologists in particular view this model 'of' human development as a cultural construction embedded in social interaction and formal institutions (e.g. education and pensions). Yet, as Holstein \& Gubrium (2007) have shown, qualitatively-informed scholars who focus on the meanings that individuals and groups attach to 'the' life course vary in the degree to which they problematize the objective 'nature' of developmental stages. Presupposing the actual existence of stages through which individuals move across biographical time, symbolic-interactionist scholars focus on 'how individuals assemble meanings and subjective understandings of everyday realities as they move through various life stations, phases, or stages' (Holstein \& Gubrium 2007, 337). In contrast, an ethnomethodologicallyinflected social constructionist approach, which we adopt here, 'concentrates on how the life course itself is constructed and used as an interpretive resource for shaping the meaning of experience in relation to time' (ibid) ${ }^{\mathrm{ii}}$. As Rosenfeld and Gallagher (2002, 359) wrote, the life course is best conceptualized as an ongoing process of 'life-coursing', which they define as 'using the typified life course as an interpretive resource for human actions and states'. Actors of varying ages conduct this interpretive work within both formal organizational settings (i.e. education and psychiatry) and less formal settings (i.e. the family and daily interactions) to assess their own and others' capacity to engage in particular actions, including those related to the management of personal and potentially stigmatizing information.

Lifecoursing is an essentially comparative process, with individuals evaluating themselves and others using 'images of growth, progress, or decline through time as guidelines for understanding or as standards for comparison (Holstein \& Gubrium 2007). Consequently, measuring discrete instances of disclosure to, for example, such members of a single (age) group as the individual's peers (e.g. friends, partners), children, or elderly parents, as does most disclosure research, obscures the lifecoursing work in which PLWH engage as they consider to whom to disclose, if at all, and, of equal importance, when in their own and in the disclosure recipient's life to do so. To expand and enrich our understanding of how age and disclosure of a stigmatized condition - here, HIV - intersect, this article explores the decision-making practices in which older PLWH 
engaged as they considered whether, and when, to disclose their HIV status to two distinct age groups within their immediate families - children and older parents.

\section{METHODS}

Recruitment, ethics, and community collaboration: The qualitative data analysed here were collected during a two-year (2011-2013), multi-method study (focus group, interview, and survey data) into the social support, mental health, and quality of life of longer-term and recently diagnosed (diagnosed for over or under ten years at the time of the study, respectively) older (aged 50+) PLWH in the United Kingdom (UK). The research was funded by the UK's Medical and Economic and Social Research Councils through their Life-long Health and Wellbeing Phase Three Pilot Study funding stream.

Of the approximately 25,000 older PLWH in the UK, most are men who have sex with men (MSM), black African heterosexual men and women, and white heterosexual men and women, in that order (see Yin et al. 2014). Accordingly, we secured a purposive sample of 123 older PLWH representing these three main groups. Of the sample of older PLWH, 23 were focus group participants, 23 completed a stand-alone mental health and quality of life survey, and 76 were interviewed and completed a mental health and quality of life survey (two of these interviews were with members of the Black Caribbean community, and were excluded from qualitative analysis). Within the sample as a whole, there were 18 black African female and 12 black African men, all of whom were heterosexual (one black African women did not declare her sexual orientation); nine white heterosexual women and one white bisexual woman; eight white heterosexual and three white bisexual men; and 50 white MSM). The age of the sample as a whole ranged from 50-87 years, but nearly all (94\%) participants were aged between 50 and 70 years.

All of the qualitative data were gathered from participants who, regardless of place of residence, accessed health or community services in London. Most of the white participants had been born in the UK, but all black African participants had been born in Africa and had migrated to the UK in their middle adulthood. Slightly more than half (52\%) of respondents were parents, but parenthood was unevenly distributed across sexual orientation, with almost all (29, or 96.7\%) of black African and four out of five (14, or $82.4 \%$ ) of white Heterosexual participants identifying as parents, versus less than a fifth (9, or 17\%) of MSM. The accounts that appear below are thus heavily weighted towards black African participants, many of whom had some or all of their children and grandchildren living in their countries of origin. Similarly, because, given the sample's age range, few participants had parents who were still living at the time the research was conducted, and many participants were diagnosed in later years, when their parents had already died, our data had fewer accounts of decisions to disclose to older parents than to younger children in later life.

After securing ethical clearance from the National Health Service (NHS) Research Ethics Committee and the study's host university, the team met with the study's Community Advisory Board. This board, composed of HIV positive advocates with an interest in HIV and aging, advised the team on ethical and effective recruitment and data collection that spoke to the concerns of PLWH. The research team then conducted three focus groups with older PLWH, recruited through and held at London-based HIV organizations. One was a mixed-gender group of black African participants (12 participants), one was a group of longer-term diagnosed PLWH (living with HIV for ten or more years) attended by MSM and heterosexual men and women of black African and white 
heritage (seven participants), and one was a group of four recently-diagnosed (living with HIV for between one and nine years) MSM. These focused discussions identified such core concerns related to living and aging with HIV as stigma, ageism, uncertainties regarding the long-term health consequences of HIV and HIV treatments, dating, disclosure, and parenting, which we built into our interview topics.

The team then conducted semi-structured life history interviews with 76 older PLWH, 74 of whom were members of our three core groups and whose interviews were subjected to qualitative analysis. Of the recently-diagnosed participants, nine were black African heterosexual men; 11 were black African heterosexual women; 22 were white MSM; seven were white heterosexual men; and five were white heterosexual women. Of the 20 longer-term diagnosed, three were black African heterosexual women, one was a black African heterosexual man, one was a white heterosexual man, three were white heterosexual women, and 11 were MSM. The research team recruited these participants through London-based HIV organizations and two HIV-dedicated clinics, and one mental health clinic serving a high number of older PLWH, in the London area. At interview, these participants were asked to describe their daily lives, social relationships, personal histories and histories with HIV, aging with HIV, quality of life, imagined futures, and HIV group attendance, if any. In keeping with our exploratory, semi-structured approach, the interviewer also asked interview participants to raise issues they felt were relevant to their experiences as older PLWH. Interviews lasted 90-120 minutes, and were recorded and transcribed verbatim by a professional transcription company before being fully anonymized (e.g. replacing participant names with numbers - see below - and changing the names of those mentioned in the data; altering or removing potentially identifying geographical and employment information; and providing pseudonyms for HIV organizations described in the data), coded, and analyzed.

Coding and Analysis: While attentive to our core research questions, our analysis was primarily responsive to themes that emerged from the data. We initially took a thematic approach to analysis (Boyatzis 1998) conducting multiple, close readings and line-by-line open coding of focus group and life-history interviews, identifying deviant cases and devising new codes as needed to accommodate variations in the data before systematizing these into a stable set of closed codes. We used these stable codes to generate folders within NVivo, into which we moved relevant sections of text. The most salient folders for the analysis presented here were comprised of segments of talk coded as 'disclosure', 'aging', 'life course', 'family', 'parenting', 'stigma', and 'HIV knowledge'.

To ensure analytic rigor, we then applied a ‘constant comparison’ method of analysis, repeatedly comparing each bit of data with other similar bits of data to develop our final analytic themes and to establish how they linked with other themes (Glaser \& Strauss 1967). Throughout all stages of analysis, we were particularly attentive to latent themes that reflected understandings, assumptions, orientations, and reasoning practices that were not explicitly articulated, but were evident across accounts and the focus group and life-history interview data. These latent understandings were what Ryan \& Bernard (2001: 91) called 'lacunae in texts [that] may indicate primal cultural assumptions' (here, those related to circumstances, capacities, needs, and trajectories seen by participants typical of particular age groups and generations). After constructing theoretical memos exploring particular findings and discussing them as a research team, the lead author searched for connections between explicit statements directly related to thematic codes (e.g. disclosure, parenting) and latent themes and searched social-scientific literatures on aging and health 
for concepts and theories through which to articulate the interpretive and decision-making practiced which our analysis had uncovered, with 'lifecoursing' (Rosenfeld and Gallagher 2002) emerging as the most conceptual framework in this regard. The authorship group debated the analysis and conceptual contribution across successive drafts to achieve inter-researcher reliability and ensure that core concepts and/or alternative explanations for our findings available in the wider aging and health literatures had not been overlooked.

To ensure that none of the quotes presented here can be traced to individual participants, we refer to interview participants by participant number, age by decade (i.e. in their 50s or 60s), and group membership (black African men as BAM, black African women as BAF; white heterosexual men as WHM, white heterosexual women as WHF; and men who have sex with men as MSM), and as recently-diagnosed (RD) or longer-term diagnosed (LTD). We refer to focus group participants by type of focus group (black African, or BA; longer-term diagnosed, or LTD; and MSM), age by decade, group membership, and recently or longer-term diagnosed, as above.

\section{STIGMA, HIV KNOWLEDGE, AND CRITERIA FOR DISCLOSURE}

Stigma: Across interviews and focus groups, participants described the continued stigmatization of HIV as the most significant factor affecting the lives of PLWH in general, including their own. Without exception, they identified HIV stigma as a barrier to their personal wellbeing, and a burden which rivalled physical ill-health in terms of its impact upon their lives ('the big burden I carry is not a health burden, it’s the stigma' - P48, a LTD WHM in her 50s). Stigma was, in these accounts, rooted in incomplete and incorrect knowledge about HIV, most notably HIV's feared casual transmissibility and its inaccurate association with other, more widely stigmatised groups (MSM, people of black African heritage, drug users, sex workers, and the promiscuous). For example, P50, a RD MSM in his 60s, said 'I know how people think: the homosexuals, people deserve it, there are African blacks, these gays ... it's the same as they see minority groups, whatever they are... I'm very aware about prejudice on the left on the right'. P47, a LTD WHF in her 60s, said that the HIVnegative, including her own friends, view PLWH as 'people who sleep around, that are drug addicts'.

HIV knowledge: Participants attributed the myths supporting HIV stigma to a lack of accurate, up to date information about the nature and transmissibility of HIV. Although participants saw various groups (for example, MSM and black Africans) as more familiar with (although not necessarily adequately knowledgeable about) the realities of HIV, given the prevalence of HIV within their communities, they characterised the HIV-negative population's knowledge of HIV as primarily if not exclusively shaped by two misleading sources. The first was the wider cultural misunderstandings of HIV and AIDS that housed the stigmatising myths detailed above. The second was the now-outdated and widely distributed public health messages of the early 1980s-1990s, which stressed the virus's sexual transmissibility and its severe, even fatal, health consequences. P5, a RD WHF in her 50s, recalled that 'HIV, years ago, when it came up, they had these horrible adverts with gravestones on, although at that point it was a terminal illness, wasn't it?' These messages had not, participants said, been followed by widely distributed public health campaigns informing the public about new developments in HIV care and the diversification of the HIV population, or seeking to combat the stigmatization of PLWH. As P77, a LTD MSM in his 50s, explained, 'the government and the NHS 
haven't kept up with the adverts, like back in the 80s ... Yes, they did the job back then, but that was for our generation. The new generation have never seen the ads'.

As a result, participants explained, knowledge about HIV was unevenly distributed across age groups. Middle and older-aged adults had been exposed to the public health messages of the 1980s1990s when they were younger, and these had shaped their understandings of HIV in middle age and into their later years. For older people, then, HIV knowledge typically consisted of outdated information encountered in the epidemic's early years and stigmatised misunderstandings embedded in the wider culture. But younger persons had not been alive at the epidemic's outset, and so had not encountered these early public health messages. For them, accurate, destigmatising HIV information was fragmented, provided (assuming that it was) in local settings (e.g. schools) embedded in a wider social context in which incorrect knowledge about HIV and HIV stigma continued to dominate. Thus, participants depicted young children as knowing little if anything about HIV, but as increasingly likely to encounter incorrect, stigmatizing messages about HIV in informal settings as they entered their teenaged years. Only in their late teens or even early adulthood, participants explained, would they learn about the realities of HIV through more trustworthy school-based sexual or health education, although even this was not assured. P61, a RD WHF in her 60s, explained, when describing her young adult son, 'I think that his age group, they have some education at school about it. I wouldn't know what that was because he'd never told me. Maybe at university or at college or something', and P1, a RD WHM in his 50s, said that his teenaged daughter was 'obviously getting sexual education at schools and things like that'. Thus, these accounts display an understanding of young adults as the age group most likely to be aware of effective treatments for HIV, and to be the least 'shocked' and 'bothered' by others' HIV status. As P72, a RD MSM in his 50s, stated when describing the MSM community: 'the young ones don't care, the middle ones are informed, the older ones are just not informed'. Similarly, P59, a RD WHF in her 60s, contrasted her young adult daughter's generation, which is 'far, far more accepting', with her own generation:

They're not shocked. In fact, you always feel they should be more worried, because they see HIV as something that's treated, like any other sexually-transmitted disease. They don't see it as being bad, or worse, or they don't see the negatives. They see a sexually-transmitted disease as being a hazard of life, if you have no steady boyfriend or girlfriend and you aren't using condoms, and HIV is just one of them. Whereas for my generation, HIV is like oh, my God!

Criteria for disclosure: Participants explained that stigmatising myths about HIV continued to threaten their own and others' social standing, life chances, and social connections and interactions, including those of great personal importance (e.g. with partners, children and parents, and friends) ${ }^{\text {iii }}$. As a result, the vast majority of participants described investing a significant amount of time, energy, and thought into maintaining control over who knew about their HIV status, and the decision to disclose as a complex one requiring careful consideration and planning. Given the potential of disclosure causing social and emotional harm to themselves and to those to whom they disclosed, participants devised and applied criteria which they felt need to be met to justify disclosing to their own children and older parents.

The first was the relevance of their HIV status to a particular individual in the context of their relationship. Participants saw sexual/romantic partners and close family as people for whom their HIV status was most likely to be relevant for purposes of safety, in the case of partners, or, in the 
case of close relatives (and particularly of parents and children, on whom we focus here) to maintain emotional closeness or the integrity of the relationship, and to provide a clear picture of their health needs in old age.

The second criterion was others' knowledge about HIV, which would affect their reaction to the disclosure. Here, an epistemological question (what do people know?) became a practical one (how do I know what this particular person knows?), with real consequences. Because those who held incorrect, and thus stigmatised, understandings about HIV were the most likely to react negatively to disclosure of HIV status, participants explained that it would be easiest, most fruitful, and least dangerous to their relationships and to their own emotional health to disclose their HIV status to those with correct knowledge of HIV. To assess specific others' knowledge about HIV, participants drew upon their actual experiences with that person and/or on their own evaluation of what this other person could be assumed to know about HIV.

The third criterion - the likelihood of the disclosure causing the disclosed-to person emotional distress - was closely related to this second criterion, as those with less knowledge of HIV were most likely to view the person disclosing his or her status to them as, e.g., highly infectious, likely to die at a relatively young age, and as promiscuous and/or a drug user, and to be emotionally distressed as a result. Another source of potential distress was the likelihood of others in the wider social setting learning of participants' HIV status and applying a 'courtesy stigma', or 'stigma by association' (Goffman 1963: 30) to children or elderly parents of PLWH, although, as we show below, this was a more pressing concern in relation to young children than it was to elderly parents.

The fourth criterion was others' ability to keep the information communicated during disclosure confidential. Participants feared that those to whom they disclosed would circulate this information to others from whom the participant had wanted to keep their HIV status private, or, at the very least, deliver the information personally. These others could react in negative ways (see above) and/or be personally distraught; moreover, they could circulate this information themselves, compounding the damage done to participants' reputations by the initial breach of confidence. Individuals to whom participants disclosed could also circulate this information to people whom the participant did not know, limiting their ability to establish new relationships on their own terms. Thus a disclosure of HIV status delivered personally and on the basis of a personal decision could be carried, through gossip, over ever widening circles, from intimates to acquaintances to strangers. Here, too, HIV knowledge was highly relevant, as, typically, those knowledgeable about the social consequences of living with HIV would be less likely to inform others of an individual's HIV status.

But the questions of whether these criteria could be met in specific instance of disclosure to children and parents could not be answered in the abstract. As we show below, participants managed this dilemma by engaging in lifecoursing: for example, evaluating whether specific children and/or older parents knew enough about HIV given their age, on the one hand, and the characteristics they considered to be typical of members of their age group, on the other.

\section{DISCLOSURE DECISION-MAKING}

Disclosure to older parents: Several participants declined to disclose to their parents or to other older relatives because they did not feel sufficiently close to them to consider their HIV status relevant to the relationship. Thus, disclosing their status would introduce a level of intimacy for which the family dynamic was unsuited. P16, an LTD MSM in his 50s, explained that his mother and family 'still don't know my diagnosis, it's got nothing to do with them'. P20, an LTD MSM in his 
60s, did not want his family to know about his HIV status 'because there’s still that barrier. I don't think they're going to be interested'.

But generating worry and upset was the most common reason provided for declining to inform parents and, sometimes, other older relatives of their HIV diagnosis (P47, a RD WHM in his 50s, hasn't 'felt the need to worry' his 97-year old father by disclosing his HIV status). P22, a RD MSM in his 50s, stated that his mother 'doesn't know' because 'it would kill her, and P57, a RD BAM in his 50s, decided that he 'wasn't going to tell my mum because she was over 70 getting to 80 then, so I said, no it would kill her'. P24, a LTD MSM in his 50s, had 'never discussed' his HIV status with his mother because

she's an 83-year-old little old lady with everything from diabetes to osteoporosis, via angina, and why should I inflict that on her? It wouldn't be fair. It doesn't make the slightest benefit for me. It doesn't make any benefit for her. It would only worry her sick. Because she's a born worrier. She worries, not only about herself but everyone who's dear to her, so we don't want to add to her long, long list of worries.

A key source of this presumed worry was a lack of correct knowledge about HIV (for example, P5, a RD WHF in her 50s, didn't want her parents or her family to know about her diagnosis 'because they're from small communities, and they wouldn't understand'). One incorrect assumption embedded within this larger lack of understanding was the now-outdated equation of HIV with poor health. P23, a RD MSM in his 50s, chose not to disclose to his mother because 'any slight cough or sneeze, it would be, are you all right? If she knew I was HIV positive, she'd be ringing up every single day, literally'. P77, a LTD MSM in his 50s, recalled telling his sister 'I would not want mum and dad to know, because the slightest thing that goes wrong they'll ultimately say is HIV related, even though mum I think is fairly knowledgeable on everything. It's still one of those things where they think, ooh, could it be? And I don't want them to keep worrying about me'.

Another incorrect assumption was the equally out-dated connection between HIV and an early and difficult death. P49, a RD MSM in his 50s, said, 'what do you think it would mean to your mum if you turn round and told her? That you're going to die an early death from AIDS; that's what she would probably anticipate'. P66, a LTD BAM in his 50s, 'couldn't even tell my mum, because the way I've seen people dying, the moment I tell her I am HIV, she will think he will die, he's going to die, that's why I took the safe way'. P44, a RD MSM in his 50s, identified his mother as someone from whom it was important to keep his HIV status secret 'because I think every parent's worst nightmare is that their children will go before them, and my mum is in her 70s now'. P23 also linked his mother's likely response to his disclosure to her outdated assumption, based on her own service to HIV/AIDS causes in the epidemic's early years, that he would die an early and 'horrific death': 'Strangely, when my brother and I came out, she also started volunteering with an AIDS charity, but again, it was when people were really ill. So, if I told her, the connection that she would make is I'm going to die within the next two years and it would be a horrific death, which it was in those days'. Thus, while he 'knew, when I was diagnosed, that things had changed dramatically in terms of treatment', P23 assumed that his mother had not kept up with these important developments, and would thus interpret his disclosure using incorrect knowledge about the health consequences of an HIV diagnosis, with emotional distress as the outcome. 
This is not to suggest that none of our participants' parents had learned of their HIV status. One participant, for example, described his mother 'picking up on' his HIV status ${ }^{\text {iv }}$, and several participants described disclosure to their older parents being 'forced', as it were, by health crises, typically those that required hospital stays ${ }^{\mathrm{v}}$. But these 'disclosures' were circumstantial rather than chosen, forced by factors that were, to a great extent, out of these participants' control: their own health visibly worsening and/or becoming so poor that their parents had to be made aware of it to maintain the participant's well-being and/or the family's close ties. In the absence of these dramatic circumstances, instances of participants choosing to disclose to their older parents were almost nonexistent.

Nor is to suggest that these older parents' knowledge of HIV was, by definition, objectively flawed. But participants overwhelmingly characterized older persons as ignorant of HIV and reliant upon stigmatizing and incorrect information about the condition, or, at best, understandings of HIV that had been correct in the epidemic's early years but were now incorrect given developments in treatment. However, while this flawed knowledge was, participants explained, characteristic of the HIV-negative world in general, older persons relied upon it to a more exclusive and extensive degree than did younger ones. This was, according to the accounts presented here, because of two factors.

The first was these elders' historical lack of exposure to changes in HIV treatment and prognosis that had emerged over the past two decades, outlined above. The second was their disengagement, through their own aging, from a world in which (a) sexual and romantic relations and activities hold a central role, and (b) they would encounter others with correct knowledge about HIV or were aware of their children's HIV status. No participant described assuming that their parents, or other older persons more widely, would gain correct knowledge of HIV as a natural feature of their future years, or would learn of their HIV from others. Rather, the working assumption evident in these accounts is that older persons, including their own parents, would retain their current (incorrect and often stigmatizing) understandings of HIV for the remainder of their lives. Here, the assumption that knowledge and, indeed, curiosity, in old age are impervious to new developments as elders disengage from the world within shrinking social domains, held in the context of a lack of public information about HIV, led participants to envisage a permanence to their older parents' incorrect understandings of HIV, and thus a certainty that disclosure would be unduly distressing, especially given their presumed isolation, lack of access to useful information or support, and increasing frailty as they aged further. This, in turn, informed participants' decision to keep their HIV status from their parents in the interests of their parents' emotional safety.

Disclosure to Children: This assumption that their older parents' worlds had shrunk and would continue to shrink, limiting both their exposure to current, accurate HIV knowledge (or to discussions of HIV in general) and their vulnerability to accidental disclosure, stands in stark contrast to participants' expectations that their own children's knowledge of HIV would expand as they grew older. These participants attributed this eventual expanded knowledge to two age-related dimensions of their children's lives. The first was their children's presumed present and future capacities: participants imagined that their children would become more socially, emotionally, and intellectually competent over time, and would become increasingly aware of, attentive to, and curious about family members, particularly when living in the same household. Both of these 'developmental outcomes' made it more likely that they would notice, correctly interpret, or seek out expressions of their parents' health status. 
The second age-related dimension was their children's structural position within educational institutions and a wider, age-stratified social world. Participants envisioned their children's eventual entrance into a world in which sexual and romantic relations held a core role; probable (although by no means assured) exposure to trustworthy education about HIV in school; and current and/or future social environments in which other children, occupying the same social space (e.g. school, play groups, and sports teams) purely by virtue of their age, could (mis)inform them about HIV and even about their parents' HIV status. Thus participants displayed an understanding of their children's future as one in which they would learn about HIV, and their parent's HIV status, 'eventually', although not necessarily from the right people or in a useful way. To these participants, this made disclosing their HIV status to their children more relevant and necessary as time went on, particularly as these eventualities existed alongside participants' concerns that their own health would decline as they aged due to their HIV status. But children's age-related susceptibilities and imagined futures also introduced dilemmas regarding the timing of disclosure within these children's own lives and the lives of participants themselves - lives which were, in the context of family relations, intermeshed.

The vast majority of participants who were parents considered their own HIV status to be highly salient to their children, linking this salience to the important role their children played in their social and emotional lives. Dedicated to protecting their children from misinformation or information about their parents' HIV status received too early in their development, participants assumed that they would need to disclose to their children 'at some point ${ }^{\text {, vi }}$. Indeed, most participants treated the relevance of their HIV status to their children as taken for granted. But relevance was seen as emergent and contingent rather than perennial, dependent upon the child's age and thus on his or her age-dependent capacity to understand the nature and implications of their parent's status. The question 'does this child need to know?' became, in practice, 'does this child need to know now, and if not, then when?' Participants who were parents identified four overlapping time frames that directed when to disclose to their children: children should be disclosed to only when they were mature enough to understand, before learning about their parents' status from others, when the disclosure would be least disruptive, and before discovering it on their own. It is important to note that these time frames did not exist in isolation. On the contrary, they often clashed, with one window opening only to be closed by another concern.

Time frames for disclosure to children: First, participants explained, children should only be informed of their parent's HIV when they were sufficiently mature to understand the information being disclosed to them, including the physical and social consequences of their parent's HIV diagnosis. These consequences included their parents' reliance on HIV medications and eventual health declining due to their HIV status, their own vulnerability to courtesy stigma ${ }^{\text {vii }}$, and the importance of maintaining their parents' confidentiality. Participants who were parents described having to balance the eventual need to tell their children about their HIV status with these children's capacity 'to understand' the nature and implications of HIV, as well as the fact that their parents had acquired HIV through sexual encounters - information which, they implied, may, depending on their children's age and maturity, be too distressing for them to learn.

P21, a LTD BAF in her 50s, discussed her HIV status with her adult children, but had not disclosed to her grandchildren, all of whom were under the age of 15 , because 'if the person is small when you tell them, maybe he won't know what you are saying'. P56, a RD WHM in his 50s, stated 
that he had disclosed his status to 'all the people that I thought were relevant and important to me' (his mother and two adult sons), and ‘now I don't tell people unless I need to tell them'. While he is 'quite open about the fact that I've got it', this is not the case 'in my house, because I've got three young children who are too young at the moment to understand'. These young children, all pre-teens, see him taking his tablets, but 'they don't know what it's for'. P45, a RD WHM in his 50s, having explained that 'it's not something you can drop into conversation with people, oh, by the way, I've got HIV', felt that he was 'getting to the stage where I would love, I might, I will, I am going to tell some people, I'm going to tell my children', as 'they're older, they're in their twenties now' and thus sufficiently mature to understand and manage the information. 'Now', he explained, 'they've both got relationships, they're adults. So I could have this conversation. And I just haven’t. My youngest was basically asking me. He said, are you happy? Because we worry about you. And I said, well, it's probably time I mentioned something to you. So it was nice to get it out'. P45 contrasted his children's current capacity to understand his disclosure with their incapacity to do so when they were younger: 'when I was diagnosed, they were obviously in their early teens, and most of all dealing with the fact that I was living in an estranged family. So I didn't feel like I needed to burden them, but if I did that they'd even understand it'. Here, his children's (past) lack of maturity, and the burdens they were facing when he was diagnosed, led P45 to delay his disclosure to them until such time as they were better prepared for it.

The maturity required of children before they were disclosed to was not only necessary for their own emotional resilience and capacity to understand. Without it, these participants explained, they would lack the discretion and self-control to keep sensitive information from others. P42, a RD WHM in his 60s, listed as one of the reasons he had not yet disclosed to his children (and grandchildren) the fact that 'they are still so young that maybe they would say something to their close friends, and I don't need that. It hasn't been much of a problem to tell them how I got it, or why, but I just don't want the people around to know, and that's why I just choose not to tell them'.

Secondly, children should be disclosed by the parent before learning about their parents' status from others, who may lack the correct HIV knowledge to provide the information appropriately and without distortion or negative evaluation. This would likely lead children to view PLWH, including their own parent, through the stigmatizing lens outlined above. As P10, a LTD BAF in her 50s, explained, without the parent using the disclosure to also educate the child about the realities of HIV, the child might draw on stigmatised constructions to think poorly of the parent: 'If before, you disclosed it and you told your children why you have it, you're okay. But if you have never told them, and then they come to know that you're HIV, a lot of people think, HIV people were like sleeping with men'. This, she says, 'puts you in a corner', an image invoked by many participants with children, who described having to time disclosure to occur when the child was sufficiently mature 'to understand', but before he or she discovered that the parent was living with HIV from others, or by 'connecting the dots' after observing the parent's health or medication use (a theme we explore below).

Another consequence of children first learning of their parents' HIV status from others was the likelihood that they would experience courtesy stigma without recourse to the help, education, and support of the parent herself. Here, participants imagine social settings into which their children were channelled as a function of their age (for example, school, play groups, and other child-specific social activities). These child-centred settings were depicted as vulnerable spaces, with children typically having little or no control over who enters them. This made children vulnerable to the 
actions and intrusions of others whose flawed (stigmatized) knowledge of HIV, and personal agendas, could be deployed in ways that could harm their children's wellbeing, sense of safety, and social position.

Without having been prepared for this stigmatization and educated as to its origins in baseless assumptions and its harmful consequences, children of PLWH would suffer from stigmatizing treatment from which their parents may not be able to protect them (if, for example, the courtesy stigma was being applied without the parent's knowledge). Thus controlling who knew about the parent's HIV status became paramount. Several participants described limiting their disclosure to a small number of people in order to keep their HIV diagnosis from a range of others who would distribute that information, through gossip, to a widening circle of people, most of whom would so lacking in correct knowledge about HIV that they would use this information to harass, bully, or frighten their child. LTFG\#1, a LTD WHM in his 50s, recalled being concerned that others' knowledge of his HIV status would affect his son, who was a child when his father was diagnosed: 'the biggest problem was for my son. I wanted to protect him at school. If somebody knew, they would be gossiping'. P42, a RD WHM in his 60s, recalled fearing that his children, who lived in 'a place where people don’t know anything about HIV, they think you get infected only to look at them', would experience courtesy stigma if their neighbours learned of his HIV status. Potential expressions of courtesy stigma, rooted in flawed conceptions of the transmissibility of HIV, included 'that your friends don't want to invite you to anything, or you have to bring your own glass, you have to have your own plate, and your own fork and knife. I've heard about it, and they had to take it home afterwards because they don't trust the dishwasher, or anything'. Because he remained 'very afraid that my kids should experience that', and believed that 'it could easily happen to my kids', P42 'chose not to tell anybody, just because of my kids. I don't trust anybody'.

In these accounts, the likelihood of participants' HIV status becoming widely known and communicated to their children, without participants' consent or provision of correct information, by people who stigmatized HIV, is so severe in its consequences that it, in itself, made disclosure to these children a pressing necessity. P56, a RD WHM in his 50s, wanted to disclose to his younger children and to educate them about the realities of living with HIV (as opposed to acquiring misinformation or HIV stereotypes). P56 anticipated that his children would face courtesy stigma, and that accurate information about HIV would enable them to cope with it: 'Obviously there's a lot of things going on with kids in schools and getting the stigma thing again, and I don't want them learning the wrong things and them getting bullied or whatever, when the time comes. Because if I give them the right information, then they should be able to cope with it a bit better'

The third time frame that directed when to disclose to their children centred on participants' belief that children should be told at times in their own lives when the disclosure would be least disruptive; for example, stressful educational demands such as studying for qualifying exams in high school or university could, participants felt, be made more stressful by the disclosure. P57, a RD BAM in his 50s, was diagnosed with HIV during the first week of his hospital stay, which lasted over a month, but waited until his son was finished with his exams before he disclosed to him. P82, a RD BAF in her 50s, had seven children, and lived with three of them: an early teenaged daughter, and two sons, both of whom were away pursuing university degrees. All of her children, except for her daughter, knew about her HIV, and her decision to disclose to her two sons was, to an extent, shaped by their own knowledge of and familiarity with HIV: 'I broke the news to the two boys, because they go for testing and they’ve got the education about HIV'. She had decided to defer 
disclosing to her daughter, however, because she is currently studying for her exams, and 'for me to tell her, I feel I might disturb her education'.

Finally, participants felt that children should learn of their parents' HIV status from their parents before discovering it on their own - through, for example, interpreting their parents' medication use or declining health as evidence of HIV. Participants often deferred disclosure to their children until such time as they felt that their own visible medication regimens, or the deterioration of their health, would force the issue. For example, P56, whose children were, he felt, too young to understand the nature and implications of his HIV were they to learn about it at their age, said that 'now they see me quite healthy, so there's no reason for them to think anything else' and that he thus envisions it being 'a year or two before I broach the subject with them'. But he also envisioned that 'they will start asking at some stage, why are you taking those tablets?' P51, a RD MSM in his 50s, had not disclosed his HIV status to his 21-year old son because 'He doesn't need to know. It doesn't improve his life. It doesn't change his and my relationship'. When he 'was ill last time', his son 'asked the question, what's happened, and why are you ill? So we just turned round and said it was a stomach problem and he's accepted that'. P51 added that if he 'became ill constantly, there would come a point where we'd have to be more forthright with him. But I don't think he needs to know at the moment'.

Other participants also envisioned a future in which their children would be faced with their declining health and/or need for help at home, in which instance they would learn of their parent's HIV status and become upset that they had not been told of it by the parent in question. Here, respondents were distressed when imagining their children facing news of their parent's HIV status, ill health, and need for care while coming to terms with their having 'kept in the dark' or even deceived. As BAFG\#9, a LTD BAF (unknown age), explained,

There is a kind of plus in disclosing to the family because as you grow older you get weaker and you need someone to give you medicine. If they find out that you have to take that medicine, and you didn't tell them about it, they will be cross with you, why didn't you tell them, 'I didn't know I had to give you that medicine' - and there will be a clash between you and them. Unless you think 100\% that you are going to be healthy throughout, I think you have to tell one or two around you.

P82, who had disclosed to her older sons but had decided not to disclose to her teenaged daughter (see above), described living with this daughter as 'a tricky situation': on the one hand, she did not want to 'disturb her education' by disclosing her HIV status, preferring to wait until 'she finishes her exams'. But 'on the other hand, also it's becoming a threat to me. You know, these younger kids, they can Google the name of your tablets. And at times, the way she asks, you think, does she know something? So it's a bit of a heartache to me sometimes'.

\section{SUMMARY AND CONCLUSION}

Our analysis of interviews and focus groups with PLWH aged 50+ shows that their decisions about disclosure were taken against the backdrop of what participants viewed as the unequal distribution of correct knowledge about HIV, and of emotional and social capacities, vulnerabilities, and skills, based, to a great degree, upon age. Because participants viewed stigmatized beliefs regarding HIV as 
originating in incorrect knowledge about the condition, they treated age as a proxy for, amongst other things, the likelihood of their disclosure producing such negative results as unwarranted confusion and 'worry', susceptibility to courtesy stigma, and disruption. Thus, when deciding whether, and when, to disclose to specific others, participants engaged in lifecoursing: here, considering these others' age and presumed circumstances, trajectories, and age-related capacities to understand the information disseminated during disclosure and the importance of keeping that information from others.

Here, then, when managing information about one's own HIV status, the age and thus the assumed capacities and circumstances of children and parents, to whom participants felt they needed to at the very least consider disclosing their status, was itself a core criterion for disclosure. In general, in regards to parents and children, the age of these other persons was a central factor in deciding whether, and when, to disclose, with these decisions based in great part on the other's perceived needs and ability to handle the news, either in general, as was the case with parents, or at that particular moment in their life, as was the case with children. Older parents' perceived inability to handle the news was informed by typified assumptions of the capacities and burdens of older people (for example, older people as subject to illness, disability, and decline and thus unable to shoulder distressing news about other people) and of members of an older generation with little correct HIV knowledge, and no experiential HIV knowledge at all. Young children were assumed to be incapable of 'handling the news' due to their ignorance of HIV, sometimes exacerbated by incorrect and stigmatising messages to which they may very well have been exposed; their inability to understand or accept their own parent's identity as sexual beings; their susceptibility to courtesy stigma and emotional upset; and their lack of the discretion and self-control to keep their parents' HIV status a purely private matter.

Participants also considered age-related typified social worlds in which children and older parents were nested. They imagined that older parents were isolated from new, accurate and timely information about HIV, and that children were forced into relatively open social settings (e.g. school, play groups) where they were subject to chance meetings with other children and even adults who had not been vetted regarding their knowledge of or attitudes to HIV, and who could confuse, misinform, exclude, or even bully these children. Thus participants considered not just the age of children and older parents as a demographic variable, but as a proxy both for age-related capacities which would wax or wane depending upon these actors' position in the life course and for these actors' position in an equally age-inflected but wider social terrain in which knowledge of HIV was unevenly distributed.

This article has uncovered the use of the life course and associated age expectations and typifications as core resources for deciding whether, and when, to disclose a stigmatized condition to those for whom the stigmatized person feels responsible and whom they want to protect (indeed, their very identities as parents and children of older parents was intimately tied to this protective ability and orientation). Because this lifecoursing activity - again, the use of age and the life course as interpretive frames - is, by its very nature, a comparative undertaking, it is less evident in research that treats age as a discrete variable, or that investigates disclosure in relation to a single age or generational group. Thus capturing the decision-making practices regarding disclosure on the part of older PLWH requires a wider lens, and more sophisticated theoretical attentiveness to comparisons and evaluations made by PLWH, than has traditionally been used in studies of aging and HIV disclosure. In short, research into the experiences of people living with HIV and other stigmatized 
conditions across the life course could benefit from a deeper sensitivity to uses of the lifecourse as interpretive resources deciding how to manage their stigmatised identities. Only through such an application can we firmly establish the adequacy of Emlet's (2008: 715) finding that 'the disclosure processes found in younger HIV-infected adults hold true for older people as well'. 


\section{REFERENCES}

Boyatzis, R.E., 1998. Transforming Qualitative Information: Thematic Analysis and Code Development. Sage.

Brown, M.J., Serovich, J.M. and Kimberly, J.A. (2016). Depressive symptoms, substance use and partner violence victimization associated with HIV disclosure among men who have sex with men. AIDS and Behavior 20(1): 184-192. doi 10.1007/s10461-015-1122-y

Cresswell, F., \& Fisher, M. (2013). HIV and the ageing patient. Medicine 41(8): 456-460.

doi:10.1016/j.mpmed.2013.05.014

Delaney, Delaney R. O., Serovich J. M. \& Lim J. (2008). Reasons for and against maternal disclosure to children and perceived child reaction. AIDS Care 20(7): 876-880. doi: 10.1080/09540120701767158

Derlega, J.V., Winstead, B.A., Greene, K., Serovich, J., and Elwood, W.N. (2002). Perceived HIVrelated stigma and HIV disclosure to relationship partners after finding out about seropositive diagnosis. Journal of Health Psychology 7:415-432. doi: 10.1080/09540120701767158.

Emlet, C.A. (2008). Truth and consequences: A qualitative exploration of HIV disclosure in older adults. AIDS Care 20(6): 710-717. doi: 10.1080/0954012070169401

Fair, C. \& Albright, J. (2012). 'Don’t tell him you have HIV unless he’s 'the one': Romantic relationships among adolescents and young adults with perinatal HIV infection. AIDS Patient Care and STDs 26(12): 746-754. doi:10.1089/apc.2012.0290.

Fernet, M., Wong, K., Richard, M.E., Otis, J., Lévy, J.J., Lapointe, N., Samson, J., Morin, G., Theriault, J. \& Trottier, G. (2011). Romantic relationships and sexual activities of the first generation of youth living with HIV since birth. AIDS Care 23(4): 393-400. doi: 10.1080/09540121.2010.51633

Garfinkel, H. (1984 [1967). Studies in Ethnomethodology. Oxford, UK: Blackwell Publishers, Inc.

Gilbert, L. and Walker, L. (2010). 'My biggest fear was that people would reject me once they knew my status...': Stigma as experienced by patients in an HIV/AIDS clinic in Johannesburg, South Africa. Health \& Social Care in the Community 18(2):139-146. doi: 10.1111/j.13652524.2009.00881.x

Glaser, B. G., and Strauss, A. (1967). The Discovery of Grounded Theory: Strategies for Qualitative Research. New York: Aldine

Goffman, E. (1963). Stigma: Notes on the Management of Spoiled Identity. Englewood Cliffs, NJ: Prentice-Hall, Inc. 
Greysen, S. R., Horwitz, L. I., Covinsky, K. E., Gordon, K., Ohl, M. E., \& Justice, A. C. (2013). Does social isolation predict hospitalization and mortality among HIV + and uninfected older veterans? Journal of the American Geriatrics Society 61(9): 1456-1463. doi: 10.1111/jgs.12410

Hockey, J. L., \& James, A. (1993). Growing Up and Growing Old: Ageing and Dependency in the Life Course. London: Sage.

Holstein, J. A., \& Gubrium, J. F. (2007). Constructionist perspectives on the life course. Sociology Compass 1(1): 335-352. doi: 10.1111/j.1751-9020.2007.00004.X

Holstein, J. A., \& Gubrium, J. F. (2000). Constructing the Life Course. Rowman \& Littlefield.

Justice, A. C. (2010). HIV and aging: Time for a new paradigm. Current HIV/AIDS Reports 7(2): 6976. doi: 10.1007/s11904-010-0041-9

Liamputtong, P. and Haritavorn, N. (2014). To tell or not to tell: Disclosure to children and family amongst Thai women living with HIV/AIDS. Health Promotion International p.dau057. First published online: July 17, 2014. doi: 10.1093/heapro/dau057

McDonald, K. (2013). Do you tell your kids? What do you tell your kids? When do you tell your kids? How do you tell your kids? HIV-positive mothers, disclosure and stigma. Pp. 129-146 in Women, Motherhood and Living with HIV/AIDS. Springer Netherlands.

Nakagawa, M.M. \& Phillips, A. (2013). Life expectancy living with HIV: Recent estimates and future implications. Current Opinion in Infectious Diseases 26(1): 17-25. doi:

10.1097/QCO.0b013e32835ba6b1.

Petrak, J.A., Doyle, A.M., Smith, A., Skinner, C. and Hedge, B., 2001. Factors associated with selfdisclosure of HIV serostatus to significant others. British Journal of Health Psychology 6(1): 69-79. doi: 10.1348/135910701169061.

Rosenfeld, D., Anderson, J., Ridge, D., Asboe, D., Catalan, J., Collins, S., Delpech, V., and Tuffrey, V. (2015). Social support, mental health, and quality of life among older people living with HIV. www.keele.ac.uk/hall.

Rosenfeld, D. and Gallagher, E.B. (2002). 'The life course as an organizing principle and a socializing resource in modern medicine'. Advances in Life Course Research 7: 357-390.

Rouleau, G., Côté, J. and Cara, C. (2012). Disclosure experience in a convenience sample of Quebec-born women living with HIV: A phenomenological study. BMC Women's Health 12(1): 37. doi: 10.1186/1472-6874-12-37.

Ryan, G.W. and Bernard, H.R. (2003). Techniques to Identify Themes. Field Methods 15(1): 85-109. doi: 10.1177/1525822X02239569 
Sankar, A., Nevedal, A., Neufeld, S., Berry, R., \& Luborsky, M. (2011). What do we know about older adults and HIV? A review of social and behavioral literature. AIDS Care 23(10): 1187-1207. doi: 10.1080/09540121.2011.564115

Schutz, A. (1964). The well-informed citizen: An essay on the social distribution of knowledge. Pp. 12-134 in Collected Papers II: Studies in Social Theory. The Hague: Martinus Nijhoff.

Shehan, C.L., Uphold, C.R., Bradshaw, P., Bender, J., Arce, N. and Bender, B. (2005). To tell or not to tell: Men's disclosure of their HIV-positive status to their mothers. Family Relations 54(2):184196. doi: 10.1111/j.0197-6664.2005.00015.x.

Smith, R. D., Kall, M. M., Rice, B. D., \& Delpech, V. C. (2011). Increasing HIV infection among adults aged 50 years and over: A call for heightened awareness. Therapy 8(2): 201-205.

doi:10.2217/thy.11.1.

Thoth, C. A., Tucker, C., Leahy, M., \& Stewart, S. M. (2014). Self-disclosure of serostatus by youth who are HIV-positive: A review. Journal of Behavioral Medicine 37(2): 276-288. doi: 10.1007/s10865-012-9485-2

van Sighem, A., Gras, L., Reiss, P., Brinkman, K., \& de Wolf, F. (2010). Life expectancy of recently diagnosed asymptomatic HIV-infected patients approaches that of uninfected individuals. Aids 24(10): 1527-1535. doi: 10.1097/QAD.0b013e32833a3946

Yin, Z., Brown, A., Hughes, G., Nardone, A., Gill, O. and Delpech, V. (2014). HIV in the United Kingdom: 2014 Report. The HIV and AIDS Reporting Team, HIV and STI Department, Centre for Infectious Disease Surveillance and Control, Health Protection Directorate.

\section{ENDNOTES}

\footnotetext{
' Dr Jane Anderson, Consultant Physician in HIV, Sexual and Reproductive Health, Homerton University Hospital NHS Foundation Trust; Dr David Asboe, Directorate of HIV Medicine and Sexual Health, Chelsea and Westminster NHS Foundation Trust; Mr Simon Collins, HIV i-Base, London.
}

ii This 'constitutive version of social constructionism' takes its theoretical lead from the works of Schutz (1964,) and Garfinkel (1984 [1967]). Schutz and Garfinkel's path-breaking work on the use of 'a stock of knowledge theoretically available to anyone, built up by practical experience, science, and technology as warranted insights' (Schutz 1964, 120) and interpretive strategies, respectively, to make sense of and reproduce everyday realities underscored the importance of treating social categories, assumptions, and practices as topics for investigation rather than as analytical resources for understanding actors' social worlds. 
iii For some participants, such fears were allayed by close others responding to news about their HIV status with empathy, or, at the very least, declarations of their continued commitment to the relationship. Almost all participants had disclosed to at least one other person, typically partners, friends, and siblings, and instances of actual rejection were few. But participants overwhelmingly characterised disclosure as a pivotal factor in their social lives, and imagined that their relationships would be ended or disrupted, and that new relationships would be cut short, should their HIV status become known (this fear was generally less pronounced, but still a major theme, in narratives of MSM participants).

${ }^{\text {iv }}$ P76, a RD MSM in his 50s, did not disclose to his mother in the months after his diagnosis, 'but she knew that something was up, she could tell, straight away. I didn't actually have to tell her, she actually guessed, which was really amazing ... I wasn't particularly good at hiding it, so she picked it up in about a couple of months'.

${ }^{\mathrm{v}} \mathrm{P} 56$, a RD WHM in his 50s, disclosed to his mother during a severe health crisis, stating that

She was really supportive. I mean, she was there all the time for me, no matter what it was, she would come to the hospital with me when I was really ill, because I was in and out, in and out, going up to the clinic every week and things like that.

P70, a RD WHF in her 50s, described an almost identical scenario in which she disclosed her HIV status to her mother when she was so ill that she had to take time off work and was so severely emaciated that her ill-health became evident. P2, a RD MSM in his 50s, had kept his HIV diagnosis from his parents because 'my partner and my ex-partner told me, oh, don't tell your parents when I got diagnosed, and I listened to them although my heart said, no, you should tell them'. But while in hospital after a drug overdose, he telephoned his parents, who 'came down and of course I wanted to tell them everything'.

${ }^{v i}$ For example, when asked who knew of his HIV, P1, a RD WHM in his 50s, answered 'My family, my children. Because they're most important to me, especially my kids'. P7, a RD BAF in her 50s, disclosed to her children 'just after my diagnosis' because 'I wanted them to know my status, there was no reason why I would hide something like that from my children'. Rare exceptions included participants with particularly contentious relationships with one or more children, as was the case with P5, a RD WHF in her 50s, who had disclosed to two of her adult daughters because 'I'm fairly close to them in a way' and 'It's important my daughters know so they know if anything happens how to deal with it as well, and also because they've got young children, protecting them against anything as well', but had not disclosed to her third adult daughter because they 'don't have a relationship'.

${ }^{\text {vii }}$ It is notable that while several participants stated that they were concerned about the prospect of their children experiencing courtesy stigma, no participants displayed similar concerns about other members of their family or, indeed, anyone else in their social worlds, although several stated that their families were worried about courtesy stigma affecting them if participants' HIV status became 
widely known. For example, LTFG\#7, a RD BAM in her 60s, who also described his sister and his daughter giving him his own cutlery and bedding out of fear that he would transmit his HIV to them, stated that 'My sister doesn't want me to let anyone know I am HIV. Family are preventing me from coming out in public. But they would probably kick me out of the house, so there are a lot of things to consider'. Here, participants note others' fears of courtesy stigma, but do not empathize with them, or proactively seek to protect them from it. 